\title{
Improved Estimation of Sway-Angle for Overhead Crane based on Phase Difference of Acoustic Signals in Frequency Domain
}

\author{
Hanako Ogawa, Takeshi Yamada, and Masayoshi Nakamoto \\ Hiroshima University, Kagamiyama 1-4-1 \\ Higashi Hiroshima, 739-8527, Japan \\ E-mail:oagwa-hanako@hiroshima-u.ac.jp,msy@hiroshima-u.ac.jp
}

\begin{abstract}
During a crane operation, swaying of the payload is an issue that should be controlled. For anti-sway control, it is essential to detect the sway angle. In this paper, we propose a method for obtaining the phase difference of the acoustic signals within the frequency domain by using the discrete Fourier transform. Based on the phase difference, we compute the time of arrival and convert it into the sway-angle of deflection. In experiment, we show the effectiveness of the proposed method by comparing with the sway angle observed from camera.
\end{abstract}

Keywords: Overhead crane, sway angle, two microphones, time difference of the arrival (TDOA)

\section{Introduction}

Cranes are widely used in industries such as factories, warehouses, and construction sites, and various types of cranes available according to their intended use. Among them, overhead cranes are frequently applied. An overhead crane carries a load using a cart on rails provided in the ceiling of the building. During the operation of the crane, there is a need for anti-swaying of the load. Anti-swaying is currently conducted manually and requires skilled workers. However, crane operators are aging, and the number of skilled operators is decreasing. Therefore, there is a growing need for computerized anti-sway control of the lifting load to support unskilled personnel, promote efficiency, and improve the safety of crane operations. With this background, research on anti-sway control is being conducted.

To conduct anti-sway control using a computer, it is essential to detect the sway angle, and an image sensor[1] or encoder[2]-[4] is mainly used. To estimate the sway angle using an image sensor, it is necessary to install the sensor at the location where the sway angle can be captured correctly. In additionFuthermore, a lengthy processing time is required to process the video signal obtained from the sensor. Direct measurements of the sway angle are difficult to achieve using an encoder when the rope of the load is deflected.
Another method for measuring the sway angle of a crane was also proposed, which uses the time difference of the arrival (TDOA) of sound waves [5]. Although with this method the sway angle is calculated from TDOA of the sound waves, the accuracy depends on the sampling frequency. In addition, with such methods, the difference in the arrival time is calculated based on the correlation; however, the computational burden is high because multiple signals are applied to find the maximum value. In addition, the experimental results also show large errors, and thus there is still room for improvement. Therefore, we propose a method for determining the phase of the acoustic signal using da discrete Fourier transformtr (DFT) and calculating the difference in the arrival time and the sway angle from the phase. We also present a frequency selection method for a sound source generation that improves the accuracy of the DFT phase calculation. There are multiple candidate values when determining the difference in the arrival time. When the frequency of the acoustic signal is low, it is more susceptible to environmental noise, and at higher frequencies, even small noise can affect the accuracy of the signal because the difference in the interval between the candidate values of the arrival time narrow despite the higher accuracy. Such aspects make it difficult to choose an appropriate difference in the arrival time. To avoid this problem, we also propose using signal processing for the correct tracking of the differences in the arrival time. 
In experiments on measuring the crane sway angle, a comparison was made between the sway angle estimated by the proposed method and that measured by the cameras. We also confirmed that the difference in the arrival time can be correctly tracked through signal processing.

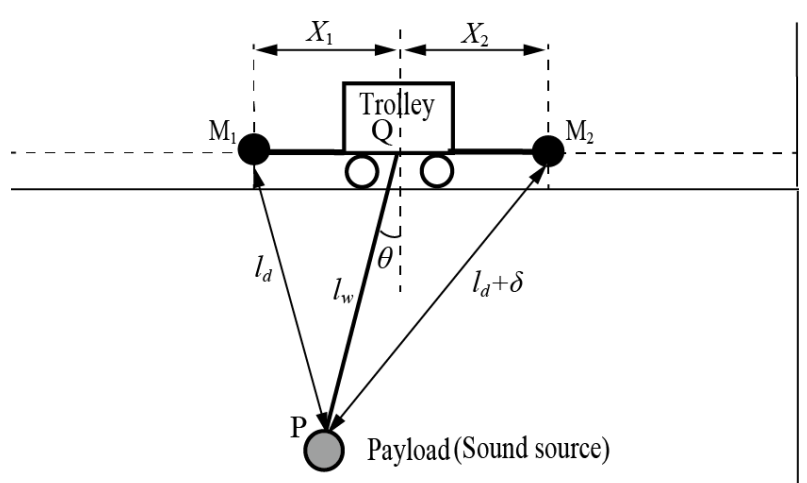

Fig. 1 Overhead crane system and its parameters

\section{Acoustic signal processing for measurement of sway angle}

In [5], two microphones are used for the contactless sensing of the angle, given the assumption that the payload (or hook) includes sound sources, as shown in Fig. 1. Using the two microphones, we estimated TDOA between the acoustic signals of the sound source in the payload.

\subsection{Estimation of TDOA}

Fig. 1 shows an overhead crane system in which the trolley has two microphones labeled $M_{1}$ and $M_{2}$. The sound source is embedded in the payload ( $P$ in Fig. 1$)$. We assume the sway angle is $\theta$, and the distance from the trolley to the sound source is $l_{w}$. Using the microphones, we first observe the sound signal originating from the sound source. It should be noted that the sensitivity to the sound signal from behind the microphones is extremely low because the microphones have directivity. Now, let $s_{1}(t)$ be the sound signal observed in $M_{1}$, and $s_{2}(t)$ be the sound signal observed in $M_{2}$.

Assume that the distance between $M_{1}$ and the trolley ( $Q$ in Fig. 1) is $X_{1}$ and the distance between $M_{2}$ and the trolley is $X_{2}$.

Hence, the distance between $M_{1}$ and the sound source is $l_{d}+\delta$, and the distance between $M_{2}$ and the sound source is $l_{d}$. Assume that $X_{1}, X_{2}$, and are known in Fig. 1. It should be noted that $\delta$ is the difference in distance between $P M_{1}$ and $P M_{2}$. Now, consider estimating $\delta$ using the TDOA. Let $\tau_{k}$ be the number of samples for the time difference between the two signals.

In [5], $\tau_{k}$ can be computed using the correlation of two signals. The TDOA between $M_{1}$ and $M_{2}$ is $\tau_{k} T_{s}$, where $T_{s}$ is the sampling period. It follows that $\delta$ can be computed as follows:

$$
\delta=\tau_{k}^{*} T_{s} v_{a}
$$

where $v_{a}$ is the velocity of sound.

The problem here is that the resolution of $\delta$ depends on $T_{s}$ because $\tau_{k} T_{s}$ are discrete values of interval $T_{s}$. Hence, we present a new approach to obtain $\delta$ that is independent of $T_{s}$. Let $\lambda_{1}(\tilde{k})$ and $\lambda_{2}(\tilde{k})$ be phases of $s_{1}(t)$ and $s_{2}(t)$, respectively. Using a discrete Fourier transform (DFT), $\lambda_{1}(\tilde{k})$ and $\lambda_{2}(\tilde{k})$ can be obtained by the following:

$$
\begin{aligned}
& S_{1}(\tilde{k})=\sum_{n=0}^{N-1} S_{1}(n) e^{-j \frac{2 \pi}{N} \tilde{k} n}=A_{1}(\tilde{k}) e^{j \lambda_{1}(\tilde{k})}, \\
& S_{2}(\tilde{k})=\sum_{n=0}^{N-1} s_{2}(n) e^{-j \frac{2 \pi}{N} \tilde{k} n}=A_{2}(\tilde{k}) e^{j \lambda_{2}(\tilde{k}),}
\end{aligned}
$$

where $\tilde{k}$ is a positive integer that corresponds to the frequency of the sound source, $N$ is the number of data, and $A_{1}(\tilde{k})$ and $A_{2}(\tilde{k})$ are the amplitudes of the frequency spectra.

Define the difference between $\lambda_{1}(\tilde{k})$ and $\lambda_{2}(\tilde{k})$ as

$$
\Delta \lambda=\lambda_{1}(\tilde{k})-\lambda_{2}(\tilde{k})
$$

Using (4), the TDOA can be computed through the following:

$$
\Delta t=\frac{\Delta \lambda}{2 \pi f_{\tilde{k}}},
$$

where $f_{\tilde{k}}$ is the frequency of the sound source. Consequently, the following velocity of sound $v_{a}, \delta$ can be obtained :

$$
\delta=v_{a} \Delta t
$$

To generate the acoustic signal of the sound source, the discrete frequency of the DFT is computed as 


$$
0, \frac{f_{s}}{N}, \frac{2 f_{s}}{N}, \ldots, \frac{(N-1) f_{s}}{N},
$$

Here $f_{s}$ is the sampling frequency $f_{s}=1 / T_{s}$. Now, it is assumed that $N$ and $f_{s}$ are given in the specifications of the hardware. In this study, the frequencies corresponding to the discrete frequencies, as indicated in (7), are used. Hence, the frequency (or frequencies) of the sound are selected such that

$$
f_{\tilde{k}}=\frac{\tilde{k} f_{s}}{N}
$$

where $1 \leq \tilde{k} \leq N-1$. That is, to achieve a high performance of (6), the frequency (or frequencies) of the sound source is decided by $N$ and $f_{s}$ of the sound receiving device.

2.2 Sway angle estimation Consider the transformation of $\delta$ to $\theta$ by the following procedure: Using the cosine theorem for $\triangle M_{1} P Q$ and $\triangle M_{2} P Q$, we have the following:

$$
\left\{\begin{array}{c}
l_{d}=X_{1}^{2}+l_{w}^{2}-2 X_{1} l_{w} \cos \left(\frac{\pi}{2}-\theta\right) \\
\left(l_{d}+\delta\right)^{2}=X_{2}^{2}+l_{w}^{2}-2 X_{2} l_{w} \cos \left(\frac{\pi}{2}+\theta\right) .
\end{array}\right.
$$

Now, letthe following:

$$
\begin{aligned}
f(\theta)= & \delta+\sqrt{X_{1}^{2}+l_{w}^{2}-2 X_{1} l_{w} \sin \theta} \\
& -\sqrt{X_{2}^{2}+l_{w}^{2}+2 X_{2} l_{w} \sin \theta}
\end{aligned}
$$

Then, $\theta$ can be obtained by computing the solution of the following equation:

$$
f(\theta)=0, \quad \theta \in\left(-\frac{\pi}{2}, \frac{\pi}{2}\right)
$$

However, it is difficult to solve (11) explicitly because lit is a non-linear equation. Hence, we introduce an algorithm to solve (11) based on Newton's method. Differentiating $f(\theta)$ by $\theta$, we have the following:

$$
\begin{aligned}
\frac{d f(\theta)}{d \theta} & =\left\{X_{1}^{2}+l_{w}^{2}-2 X_{1} l_{w} \sin \theta\right\}^{-\frac{1}{2}}\left\{-X_{1} l_{w} \cos \theta\right\} \\
& -\left\{X_{2}^{2}+l_{w}^{2}+2 X_{2} l_{w} \sin \theta\right\}^{-\frac{1}{2}}\left\{X_{2} l_{w} \cos \theta\right\} .
\end{aligned}
$$

Let $\theta_{0}$ be an initial value of the sway angle, and $\theta_{n}$ be the sway angle at the $n$-th step. It follows from (12) that Newton's method can be used to solve (11). Thus, we obtain the sway angle by repeating the following procedure:

$$
\theta_{n+1}=\theta_{n}-\frac{f\left(\theta_{n}\right)}{\left\{\frac{d f\left(\theta_{n}\right)}{d \theta}\right\}} .
$$

The terminal condition is $\left|\theta_{n+1}-\theta_{n}\right|<\varepsilon$ wwhere $\varepsilon$ is a small positive number.
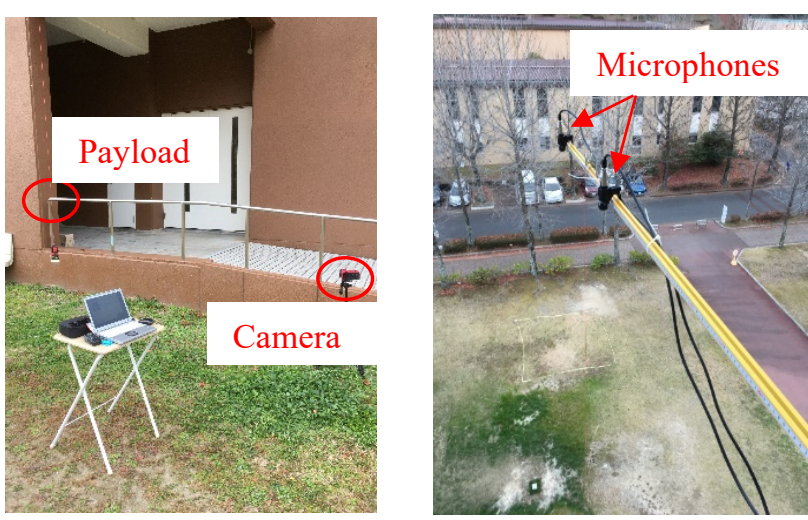

Fig. 2 Experimental devices (microphones, payload, camera)

\section{Experiment}

Fig. 2 shows the experimental equipment. In this measurement, a sine wave was used as the sound source in the payload. Here, the parameter of the terminal condition is set as $\varepsilon=0.0001$. The parameters are $l_{w}=$ $15.11[\mathrm{~m}], N=10^{2}, f_{s}=44100[\mathrm{~Hz}]$, and $X_{1}=X_{2}=$ 30 [cm]. In addition, video clips were captured, and the sway angles were then calculated from the image processing and compared. The frequency spectra of the observed signals are shown in Fig. 3. In this experiment, the frequencies of $f_{k}=5038.77[\mathrm{~Hz}]$ and $f_{k}=$ 8010.35 [Hz] were used.

The payload (sound source) was individually vibrated. The estimation of $\theta$ for $f_{k}=5038.77[\mathrm{~Hz}]$ is shown in Fig. 4 where an offset of stationary state is considered. Similar to Fig. 4, the estimation of $\theta$ for $f_{k}=$ $8010.35[\mathrm{~Hz}]$ is shown in Fig. 5. The blue line is the 

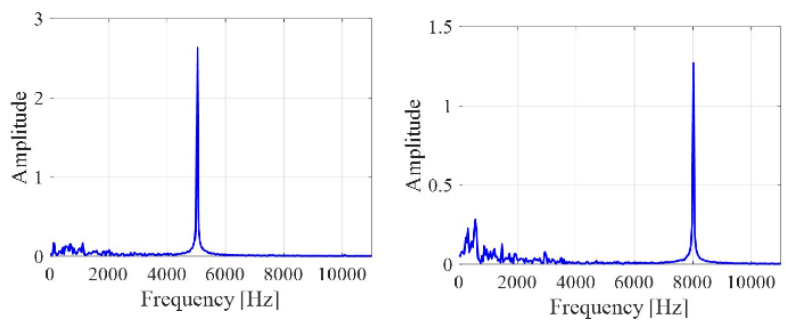

Fig. 3 Frequency spectra of observed signals

estimated sway angle from the sound (proposed method), and the red line is the sway angle from the image processing.

Fig. 5 shows that the sway angle of the proposed method agrees well with the sway angle of the image processing, and it can be confirmed that the quality point tracking can be achieved even under the influence of disturbances and noises on the sound source from a blower. Comparing Figs. 4 and 5, we can see that the result in Fig. 5 has a smaller error. As the reason for this, the frequency bands of the blower noise do not overlap with the frequency bands of the sound source. That is, the deflection angle can be accurately estimated, which confirms the effectiveness of the proposed method.

\section{Conclusion}

We considered a soft sensor to measure the sway angle for the anti-sway control of an overhead crane. We proposed an approach to estimate the angle based on acoustic signals to achieve contactless sensing of the angle. The method uses the phase difference of acoustic signals measured by two microphones. We presented an algorithm to obtain the angle from the phase difference based on Newton's method. During the experiment, we compared the proposed method with measurements taken from photographs. As a result, it was confirmed that the proposed method can dynamically follow the sway angle of the payload. Furthermore, we confirmed that the proposed method can correctly track the sway angle.

\section{References}

1. C.-Y. Chang and H. W. Lie: Real-Time Visual Tracking and Measurement to Control Fast Dynamics of Overhead Cranes, IEEE Trans. Ind. Electron., vol.59, no.3, pp.16401649, March 2012.

2. B. Lu, Y. Fang, N. Sun and X. Wang: Antiswing Control of Offshore Boom Cranes With Ship Roll Disturbances, IEEE Trans. Control Systems Technology, vol.26, no.2, pp.740-747, Mar. 2018.
3. N. Sun, T. Yang, H. Chen and Y. Fang: Dynamic Feedback Antiswing Control of Shipboard Cranes Without Velocity Measurement: Theory and Hardware Experiments, IEEE Trans. Ind. Inform., vol.15, no.5, pp.2879-2891, May 2019.

4. N. Sun, Y. Fu, T. Yang, J. Zhang, Y. Fang and X. Xin: Nonlinear Motion Control of Complicated Dual Rotary Crane Systems Without Velocity Feedback: Design, Analysis, and Hardware Experiments, IEEE Trans. Automation Science and Engineering, vol.17, no.2, pp.1017-1029, April 2020.

5. M.Matsunaga, M.Nakamoto and T.Yamamoto: A soundbased measurement of sway angle for anti-sway control of over head crane, Journal of Robotics, Networking and Artificial Life, Vol.4, No.4, pp.322-355, March 2018.

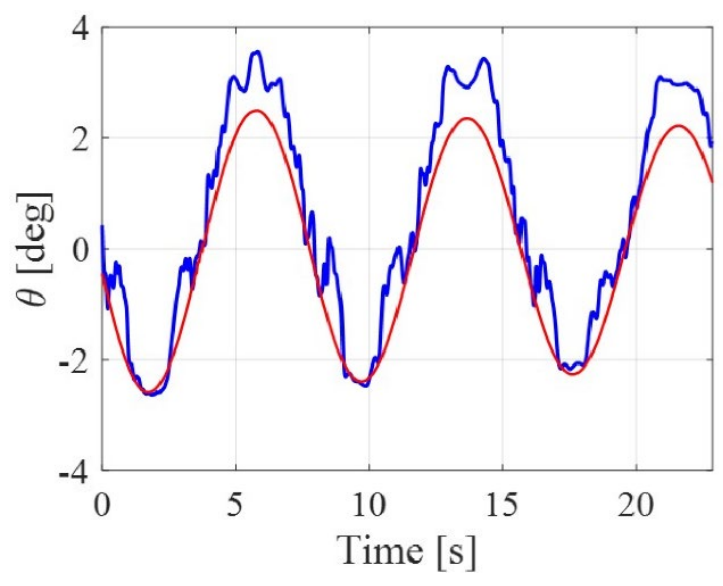

Fig. 4 Results of estimating $\theta$ for $f_{k}=5038.77[\mathrm{~Hz}]$

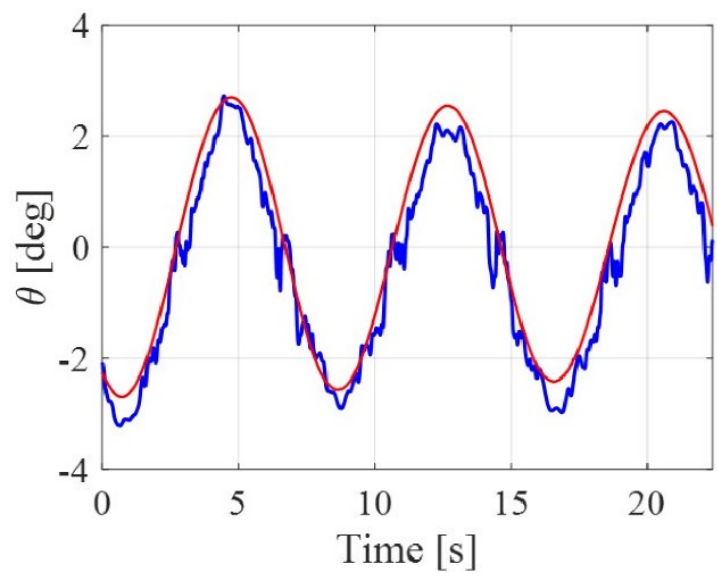

Fig. 5 Result of estimating $\theta$ for $f_{k}=8010.35[\mathrm{~Hz}]$ 